\title{
Oral Microbiota Change in Intubated Patients under Mechanical Ventilation
}

\author{
Yoon Hee Choi' ${ }^{1}$, Soo Hyun Kim², Yeuni Yu ${ }^{3}$, Seo-Young Lee ${ }^{3}$, Yu Jung Jung ${ }^{4}$, Do Young Kim ${ }^{4}$, \\ Myoung Soo Kim ${ }^{5}$, Hee Sam $\mathrm{Na}^{6 *}$
}

1'Department of Internal Medicine, Dongnam Institute of Radiological and Medical Sciences, Busan 46033, Republic of Korea

${ }^{2}$ Artificial Kidney Room, Busan Medical Center, Busan 47527, Republic of Korea

${ }^{3}$ Interdisplinary Program of Genomic Science, Pusan National University, Busan 46241, Republic of Korea

${ }^{4}$ School of Dentistry, Pusan National University, Yangsan 50612, Republic of Korea

${ }^{5}$ Department of Nursing, College of Natural Science, Pukyong National University, Busan 48513, Republic of Korea

${ }^{6}$ Department of Oral Microbiology, BK21 PLUS Project, School of Dentistry, Pusan National University, Yangsan 50612 ,

Republic of Korea

\author{
Corresponding \\ Hee Sam Na, Professor \\ Department of Oral Microbiology, School \\ of Dentistry, Pusan National University, \\ Busandaehak-ro 49, Mulgeum-eup, \\ Yangsan, Gyeongsangnam-do 50612, \\ Republic of Korea \\ Phone : +82-51-510-8252 \\ Fax : +82-51-510-8246 \\ E-mail : heesamy@pusan.ac.kr
}

Received : September 3, 2021

Revised : September 27, 2021

Accepted : September 27, 2021

No potential conflict of interest relevant to this article was reported.

Copyright (C) 2021 Journal of Bacteriology and Virology

(C)This is an Open Access article distributed under the terms of the Creative Commons Attribution Non-Commercial License

(http://creativecommons.org/

license/by-nc/3.0/).
One of the complications that may develop in intubated patients under mechanical ventilator is ventilator-associated pneumonia (VAP). In most cases, VAP appears to result from aspiration of the oropharyngeal microflora or colonizing pathogens. For efficient prevention and management, understanding oral microbiome dynamics during mechanical ventilator is required. In this study, we analyzed longitudinal oral microbiome in intubated patients under mechanical ventilation support. Intubated patients and healthy subjects were recruited for this study. Buccal samples were collected for microbiome study. For longitudinal comparison, intubated samples were grouped as initial 2 days, first week, second week, third week, and over 3 week group. 16S ribosomal RNA next generation sequencing was applied for microbiome analysis. Alpha diversity and beta diversity was applied to characterize the oral microbiome. Linear discriminant analysis effect size (LefSe) was applied to determine difference in the composition among tested group. In intubated patients, the richness and evenness of the microbiota was significantly reduced compared to the healthy control. The most abundant species in intubated patients were Corynebacterium, Acinetobacter, Streptococcus, Staphylococcus, and Enterococcus, which represented more than $70 \%$ of the total sequences at the genus level. The most abundant species were Acinetobacter baumannii, Proteus mirabilis, Enterococcus faecalis Pseudomonas aeruginosa, and Gardnerella vaginalis were among the significant taxa within intubated patients. Pathogenic bacteria including Acinetobacter, Enterococcus, Klebsiella, Pseudomonas, and Staphylococcus were also frequently observed during the observation period. In intubated patients, the composition of oral microbiome was heavily distorted compared to healthy subjects and high abundance of pathogenic bacteria was observed. A better understanding of the longitudinal microbiome change in oral cavity of intubated patients should provide better understanding for effective prophylactic and therapeutic interventions for VAP.

Key Words: Oral microbiota, Intubation, Mechanical ventilator, Ventilator associated pneumonia 


\section{INTRODUCTION}

One of the complications that may develop in ventilated patients is ventilator-associated pneumonia (VAP), which contributes to approximately half of all cases of hospital-associated pneumonia and is the second most common nosocomial infection in the intensive care unit (ICU) (1). Several different routes of infection by VAP pathogens have been proposed, but in most cases infection appears to result from aspiration of the oropharyngeal microflora or colonizing pathogens (2). Intubation and critical illness reduce oral immunity and can exacerbate poor oral hygiene $(3,4)$. Dental plaque accumulates rapidly in the mouths of critically ill patients and colonization by microbial pathogens is likely as the amount of plaque increases $(5,6)$. Microaspiration from oropharyngeal microbes are thought to play a role in the etiology of VAP (7) and the high prevalence of oral colonization with VAP-associated pathogens have been reported (8). VAP is often a clinical diagnosis without confirmation by bacterial culturing and subsequent identification of putative pathogens (9). In a study of mechanically ventilated patients, $80 \%$ of culture-negative respiratory samples had high abundances of oral bacteria (10). Several studies have shown that maintenance of oral hygiene in patients under mechanical ventilator can reduce the rate of VAP $(11,12)$. Thus, understanding the oral microbiome dynamics in intubated patients should help prevent or manage VAP in ICU patients.

$16 \mathrm{~S}$ ribosomal RNA (rRNA) gene sequencing has allowed culture-independent characterization of the bacterial communities in health and disease (13-15). Although oral microbiome has been extensively studied in oral diseases such as periodontitis and caries, it has not been extensively studied in intubated subjects under mechanical ventilator. Here, we performed serial $16 \mathrm{~S}$ rRNA gene sequencing of buccal samples from intubated patients under mechanical ventilator. Our goals were to identify features of bacterial community structure in intubated subjects and characterize the dynamics of the oral bacterial microbiome during mechanical ventilation.

\section{MATERIAL AND METHODS}

\section{Participants}

The inclusion criteria for ICU patients were; 1) $>18$ years of age, 2) endotracheal tube use with holding methods, 3) consent for participation from the patient's family. For healthy control, age matched 13 volunteers ( 7 male, 6 female, age $67.5 \pm 7.4$ ) without any systemic diseases were recruited. The enrolled intubated patients ( 9 male, 1 female, age $68.8 \pm$ 11.2) include a heterogeneous set of underlying diseases (Table 1). Average observation period was $10.7 \pm 12.4$ days (range: 1-41 days) and $7.9 \pm 6.9$ (range :1-21) samples per patients were collected. Broad spectrum antibiotics were

Table 1. Characterization of subjects under mechanical ventilation

\begin{tabular}{cccccc}
\hline Subject & Age & Sex & Underlying disease & Initial Antibiotics & Days observed \\
\hline 1 & 81 & F & DM, Parkinsonism & Piperacillin-Tazobactam & 41 \\
2 & 71 & M & Chronic kidney disease, Intracerebral hemorrhage & Ceftriaxone & 1 \\
3 & 81 & M & COPD, Alcoholic hepatitis, DM & Piperacillin-Tazobactam & 12 \\
4 & 52 & M & Ceberal Palsy & Piperacillin-Tazobactam & 6 \\
5 & 85 & M & COPD & Meropenem Colistin & 5 \\
6 & 71 & M & Lung Cancer RCC & Meropenem & 13 \\
7 & 57 & M & Encephalitis & Ertapenem & 24 \\
8 & 67 & M & Alcoholic Hepatitis & Piperacillin-Tazobactam & 10 \\
9 & 65 & M & DM, COPD, R/O Asthna & Cefriaxone & 1 \\
10 & 58 & M & DM, Liver cirrhosis, hypothyroidism & &
\end{tabular}


administered to the patients. Carbapenem (7/10) which include ertapenem and meropenem was most frequently prescribed antibiotics followed by penicillin (6/10) including piperacillin-tazobactam (Table 1). For longitudinal microbiome analysis, several timepoints were selected as follows; initial samples collected within 2 days (Day2), $1^{\text {st }}$ week samples (1 $w k), 2^{\text {nd }}$ week samples ( $2 w k$ ), $3^{\text {rd }}$ week samples ( $3 w k$ ) and samples collected over 3 weeks (over $3 w k$ ).

This study was approved by the Institutional Review Board (DAUHIRB-19-072). Intubated participants in this study were mostly sedated or at severely poor levels of consciousness; therefore, family members were considered as the legal representatives with the power of attorney based on the International Council for Harmonization (16). During the participant recruitment period, the purpose, voluntary nature of participation, confidentiality of information, and procedures used in the study were explained to family members, and informed consent was obtained from each family.

\section{Oral swab sample preparation}

Buccal swap samples were collected using the Levine technique; the swab was rotated over an approximately $1 \mathrm{~cm}^{2}$ area with sufficient pressure (17). Sterile cotton swabs were used for the collection and were placed in a $15 \mathrm{~mL}$ conical tube labelled with the sample ID and stored at $-80^{\circ} \mathrm{C}$.

\section{Extraction of Genomic DNA and Next Generation Sequencing}

Samples were suspended in phosphate buffered saline (PBS), vortexed, and then centrifuged. DNA was extracted using a Gram positive DNA purification kit (Lucigen, Biosearch Technology, Novato, CA) following the manufacturer's instructions. The final concentration was measured with a NanoDrop ND-1000 spectrophotometer (Thermo Fisher Scientific, USA) and stored at $-80^{\circ} \mathrm{C}$ until use. PCR amplification of the $16 \mathrm{~S}$ ribosomal RNA gene V3-V4 region (341F-785R) was performed. The primer sequences used for amplifications were as follows: 341F: 5'-CCT ACG GGN GGC WGC AG-3', 785R: 5'-GAC TAC HVG GGT ATC TAA TCC-3'(18). Purified amplicons were pooled in equimolar and paired-end sequenced with MiSeq (Illumina, San Diago, CA, USA).

\section{Bioinformatic Analysis, Statistical Analysis, and Visualization}

Basic microbiome analyses have been performed using the QIIME2 (version 2020.6) (19) and associated plugins. To measure alpha diversities, Faith PD index, Choa1 index, and Shannon's index method were used. For the beta diversity analysis, the weighted UniFrac principle coordinates analysis ( $P C O A)$ was used. The Kruskal-Wallis test was used to assess the statistical significances between the groups for alpha diversity and relative abundance. To assign taxonomy to the unique representative sequences, pre-trained Naive Bayes classifier, using Human Oral Microbiome Database (eHOMD) 16S rRNA Extended RefSeq sequences (version 15.1) (20), were used. To test differential abundance of bacterial species among test groups, linear discriminant analysis effect size (LEfSe) (21) was applied with default settings.

\section{RESULTS}

\section{Diversity and abundance of microbiota}

The alpha diversity of the microbiota was estimated by Chao1 (Fig. 1A), Faith PD (Fig. 1B), and Shannon test (Fig. 1C). In intubated patients under mechanical ventilator, richness (Chao1 and Faith PD) of the microbiota was significantly reduced compared to the healthy subjects (Fig. 1A and 1B). The evenness (Shannon) of intubated patients were reduced compared to the healthy subjects but statistical significance was not prominent (Fig. 1C). To visualize the overall microbiome structure, weighted UniFrac PCOA was applied. The microbiome composition was significantly different between healthy subjects and intubated patients (Fig. 1D). 
(A)

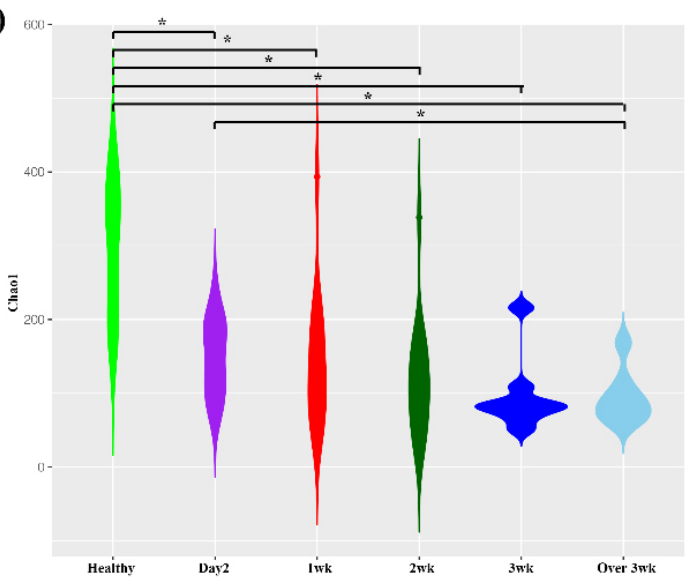

(C)

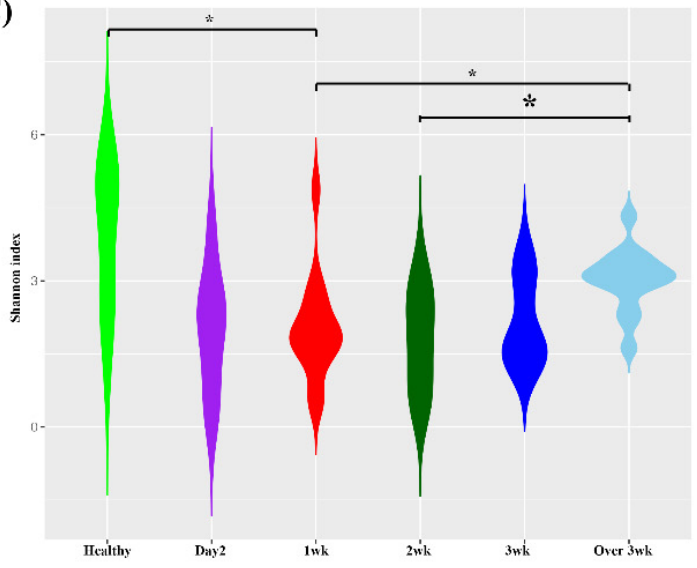

(B)

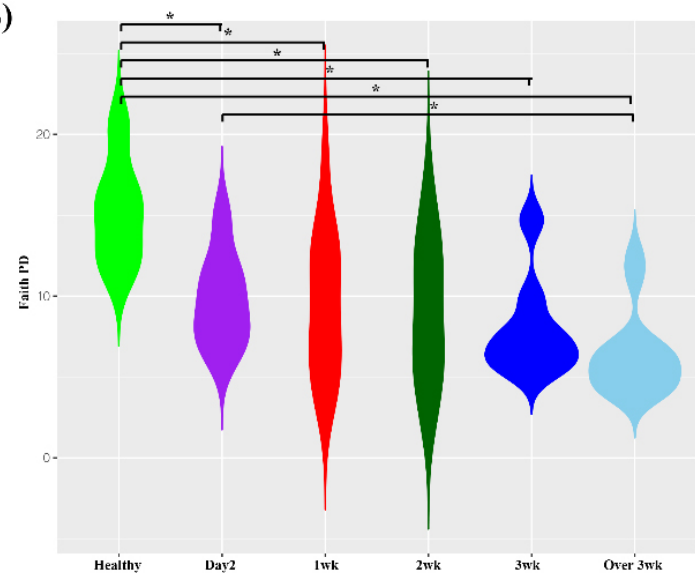

(D)

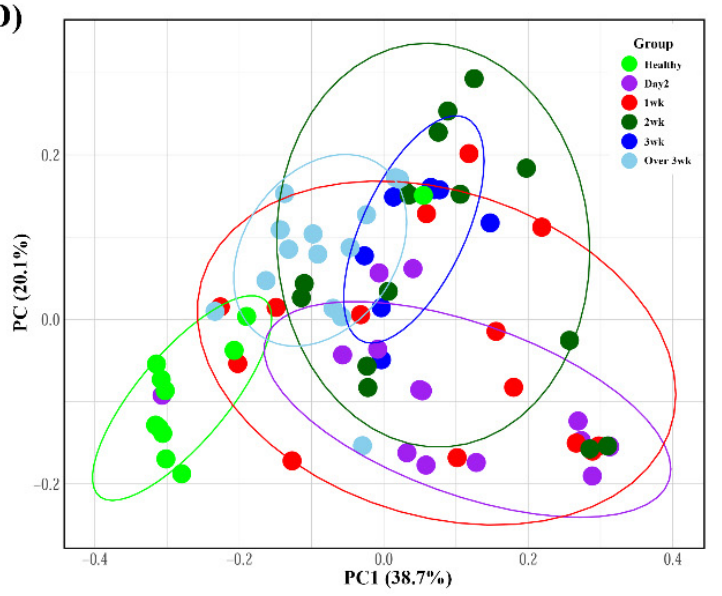

Fig. 1. Bacterial community comparisons. Buccal samples were collected from healthy and intubated patients. (A) Chao1 alpha diversity (B) Faith's PD alpha diversity (C) Shannon alpha diversity, (D) Weighted UniFrac Principle Coordinate beta diversity. $\star p<0.05$

Next, abundance of the microbiota was analyzed. At the phyla level, the five most abundant were Firmicutes, Proteobacteria, Bacteroidetes, Actinobacteria, and Fusobacteria, which represented more than $99 \%$ of the total sequences. The abundance of Actinobacteria was most abundant in intubated patients within 2 days and their abundance showed gradual decrease. Compared to healthy subjects, the abundance of Bacteroidetes and Firmicutes was decreased in intubated patients during the treatment (Fig. 2A). At the genus level, the most abundant genera in the healthy subjects were Streptococcus, Gemella, Neissseria, Heamophilus, and Klebsiella, which constituted more than $70 \%$ of the total sequences. In intubated patients, Corynebacterium, Acinetobacter, Streptococcus, Staphylococcus, and Enterococcus represented more than $70 \%$ of the total sequences. In intubated patients, the abundance of Corynebacterium and Enterococcus was most abundant in day 2 group and showed gradual decrease while the abundance of Acinetobacter and Staphylococcus showed gradual increase from initial treatment to week 3 (Fig. 2B).

\section{Species taxa comparison}

LEfSe was applied to evaluate the differences in bacterial abundance during ventilator treatment in incubated patients. In initial day2 samples, the abundance of Corynebacterium, Veillonella dispar, Prevotella, and Campylobacter gracilis were significantly higher compared to other time-points. In $1^{\text {st }}$ week samples, the abundance of Campylobacter, Actinomyces, 
and Capnocytophaga were significantly higher compared to other time-points. In $3^{\text {rd }}$ week samples, the abundance of Acinetobacter baumannii, Staphylococcus, Proteus mirabilis, and Enterococcus faecalis were significantly higher compared to other time-points. In over 3 week samples, the abundance of Pseudomonas aeruginosa, Corynebacterium amycolatum, Tannerella forsythia, and Gardnerella vaginalis were significantly higher compared to other time-points (Fig. 3A and 3B). Finally, the abundance of significant species identified by LEfSe was validated. The abundance of Lautropia mirabilis,
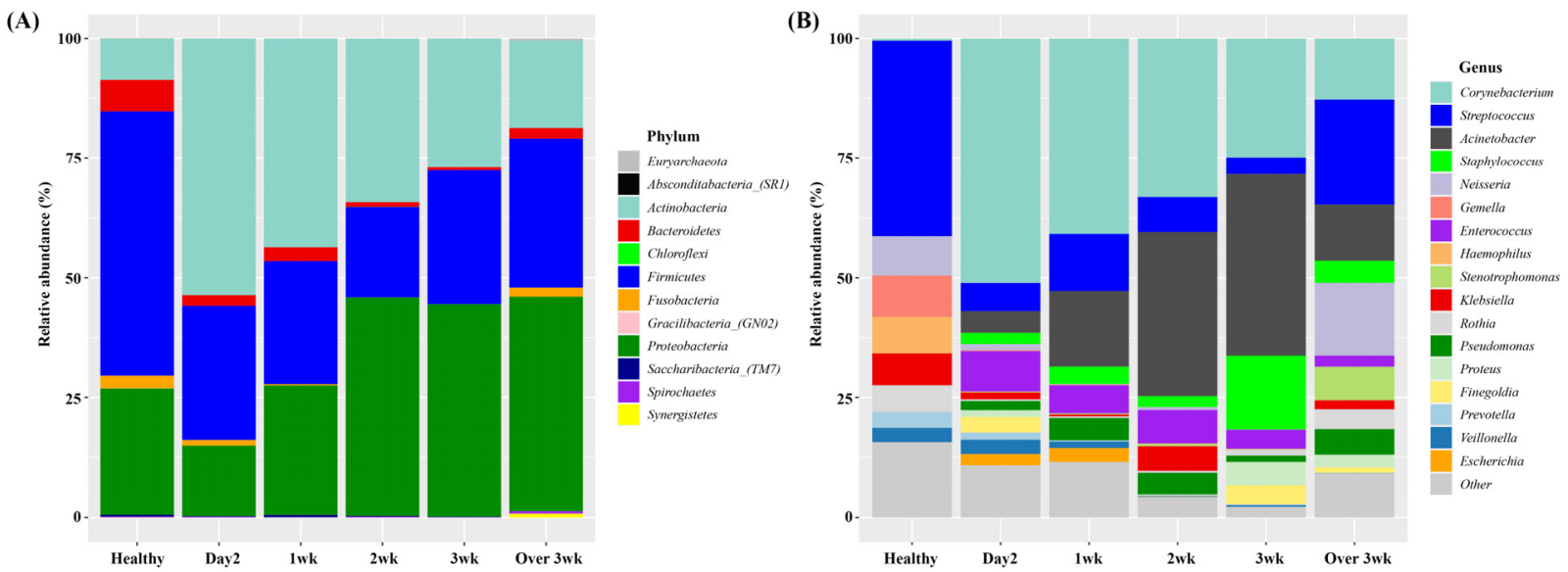

Fig. 2. Relative abundance of bacterial species in healthy and intubated patients at various time points. (A) Phylum level and (B) Genus level. Day2: samples collected within initial 2 days intubated, 1 wk: samples collected within first week, 2 wk : samples collected within second week, 3 wk: samples collected within third week, over 3 wk : samples collected over 3 week.

(A)

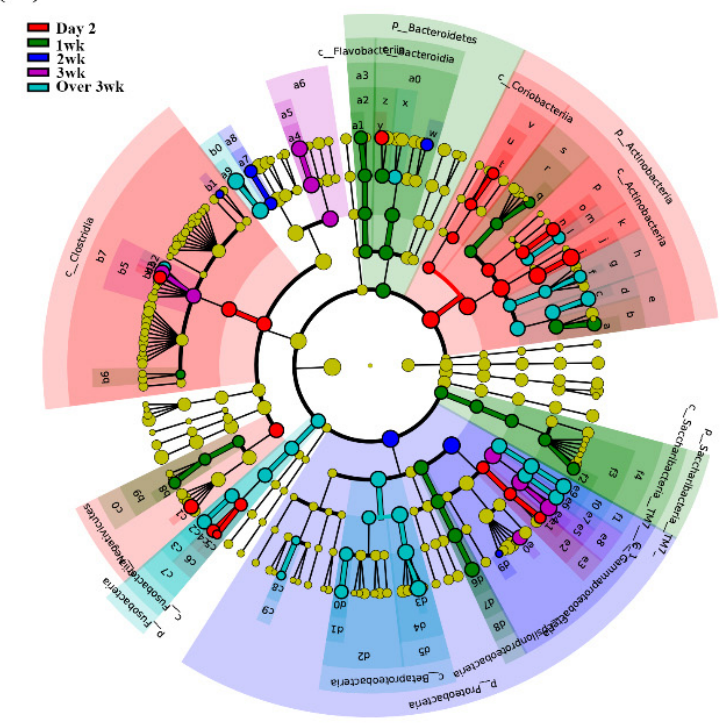

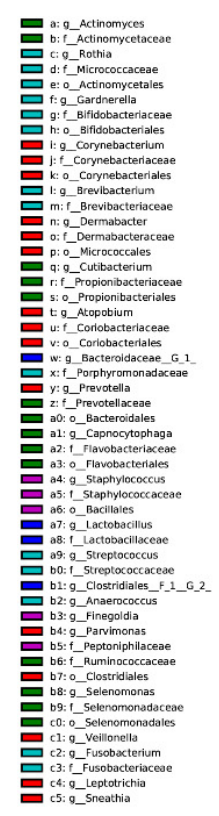

(B)

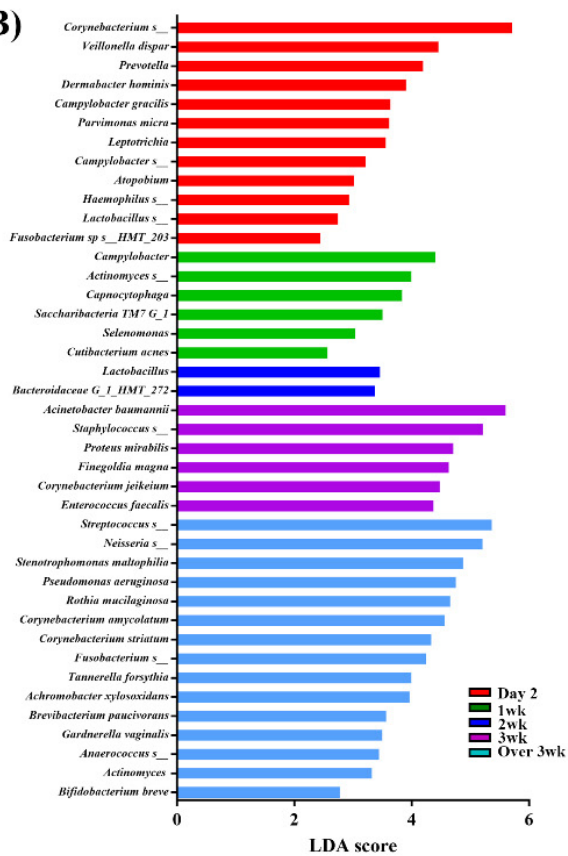

Fig. 3. Comparisons of microbiota depending on intubation period that presented significantly different contents. (A) Cladogram of significant species. (B) Linear discriminant analysis (LDA) score of significant species. The analysis has been performed using linear discriminant analysis effect size (LefSe) method. Day2: samples collected within initial 2 days intubated, 1 wk: samples collected within first week, 2 wk: samples collected within second week, 3 wk: samples collected within third week, over 3 wk : samples collected over 3 week. 

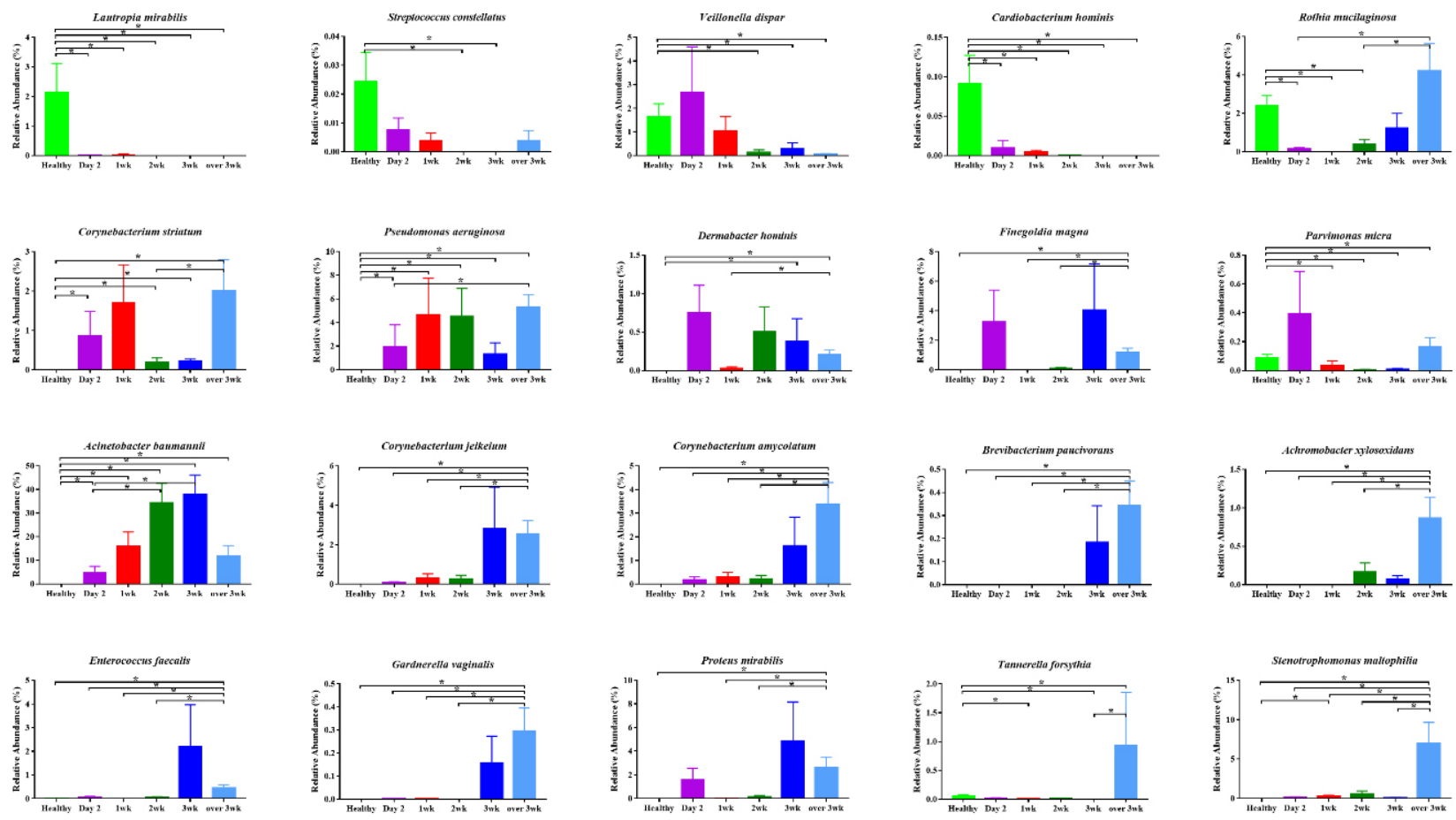

Fig. 4. Relative abundance of selected significant bacterial species. The difference between groups was assessed using Kruskal-Wallis test. * indicates a $p$-value less than 0.05 .

Streptococcus constellatus, and Cardiobacterium hominis was abundant in healthy subjects and showed abrupt decreased following intubation. The abundance of Acinetobacter baumannii showed gradual increase from day 2 to week 3 in intubated patients. Several bacteria showed significant increase in samples over 2 weeks (week 3 and over 3 week samples), e.g. Corynebacterium jeikeium, C. amycolatum, Brevibacterium paucivarans, Achromobacter xylosoxidans, Enterococcus faecalis, Gardnerella vaginalis, Proteus mirabilis, Tannerella forsythia, and Stenotrophomonas maltophila (Fig. 4).

\section{DISCUSSION}

Intubation and the presence of the endotracheal tube can exacerbate poor oral hygiene in ICU patients receiving mechanical ventilation treatment $(3,4)$. Pulmonary complications of mechanical ventilation include VAP (22). Respiratory pathogens can colonize the oral cavity prior to entering the lower respiratory tract (23). For efficient VAP prevention and management, it is imperative to understand the features of bacterial community structure and the dynamics of the oral bacterial microbiome during mechanical ventilation. In this study, we analyzed longitudinal oral microbiome change to understand the microbiome dynamics in intubated patients.

To characterize the initial oral microbiome change in intubated patients, healthy participants were recruited for comparison. Since oral microbiome dynamically change during mechanical ventilation, several time points were selected for the analysis. Compared to healthy subjects, the richness (Chao1 and Faith PD) and evenness (Shannon index) of the microbiota was significantly decreased in intubated patients. A course of antibiotics can lead to a marked reduction in species diversity and expansion of relative abundance of certain taxa (24). Since most of the patients enrolled in this study had been on antibiotic therapy, effect of antibiotics could have influenced the oral microbiome diversity. Also when beta-diversity was determined, the oral microbial structure was significantly different between healthy subjects and intubated patients suggesting the oral microbiome structure was significantly distorted in intubated patients. 
When overall abundance was determined, Corynebacterium, Acinetobacter, Streptococcus, Staphylococcus, and Enterococcus represented more than $70 \%$ of the total sequences in samples from intubated patients. The abundance of Corynebacterium was most abundant in Day2 samples and remained highly abundant in intubated patients during the observation. The relative abundance of the genus Corynebacterium have been reported to be significantly higher in the pneumonia compared to healthy subjects (25). At the species level, the overall microbiome structure of intubated patients was significantly different from healthy subjects. When LEfSe was applied to compare significant taxa between healthy and intubated patients, a very long list of bacteria taxa was produced. Thus, we focused significant taxa within intubated patients. At each time point, there were several bacteria taxa significantly increased compared to other time points. The abundance of Veillonella dispar, Campylobacter gracilis, Acinetobacter baumannii, Proteus mirabilis, Enterococcus faecalis Pseudomonas aeruginosa, Corynebacterium amycolatum, Tannerella forsythia, and Gardnerella vaginalis were among the significant taxa in intubated patients. A. baumannii is an opportunistic human pathogen that predominantly infects critically ill patients, which causes a range of nosocomial infections across multiple anatomical sites and most commonly it manifest as ventilator-associated pneumonia or central line-associated blood stream infections (26). Especially, Acinetobacter have been noted to be a primary pathogen of VAP in some Asian countries $(27,28)$.

During longitudinal observation, pathogenic bacteria including $A$. baumanni, Enterococcus, Klebsiella pneumoniae, Pseudomonas aeruginosa, and Staphylococcus aureus were also frequently found. In a meta-study that included 24 investigations conducted with ventilated patients, gram negative bacteria represented $58 \%$ of recovered organisms in VAP patients. The predominant gram negative bacteria were $P$. aeruginosa and Acinetobacter spp., followed by Proteus spp., Escherichia coli, Klebsiella spp., and H. influenzae. Among gram positive bacteria, S. aureus was involved in $20 \%$ of the cases (22). Moreover, known VAP pathogens such as $H$. influenzae, Escherichia Sp., S. pneumoniae, Proteus mirabilis, and Pseudomonas sp. have been identified in the oral cavity from patients with VAP (23). Thus, strong coincidence of pathogens in oral cavity and VAP supports the need to monitor oral microbiome to prevent or manage VAP in intubated patients.

When the microbiome change of the significant taxa was validated, oral microbiome commonly found in healthy subjects showed abrupt decrease in intubated patients. The abundance of $A$. baumannii showed gradual increase in intubated patients and there were some bacteria that showed significant increase in samples over 2 weeks (week 3 and over 3 week samples). Thus, there is continuous oral microbiome change in intubated patients. There are several limitations to this study design. First, the study uses a relatively small sample size. A limited number of observable patients were available for participation, because intubated patients usually underwent a tracheostomy within 2 weeks, and even more critical patients were directly selected for tracheostomy. Our findings should be validated in a larger clinical cohort. Although power calculations were not feasible in this study, an adequate number of participants would be needed to allow stratification of participants into different predisposing medical conditions or antibiotics. Second, a well-designed longitudinal study with adequate sample size is needed to elucidate the role of oral microbiome in VAP development or clinical outcome.

The present study provides valuable information concerning the oral microbiome in intubated patients. A better understanding of the sequential microbiome change in oral cavity of intubated patients and the role of oral bacteria in VAP development should provide better understanding for effective prophylactic and therapeutic interventions for VAP.

\section{ACKNOWLEDGEMENTS}

This work was supported by a 2-year research grant of Pusan National University. 


\section{REFERENCES}

1) Joseph NM, Sistla S, Dutta TK, Badhe AS, Parija SC. Ventilator-associated pneumonia: a review. Eur J Intern Med 2010:21:360-8.

2) Shi Z, Xie H, Wang P, Zhang Q, Wu Y, Chen E, et al. Oral hygiene care for critically ill patients to prevent ventilatorassociated pneumonia. Cochrane Database Syst Rev 2013:CD008367.

3) Labeau SO, Van de Vyver K, Brusselaers N, Vogelaers D, Blot SI. Prevention of ventilator-associated pneumonia with oral antiseptics: a systematic review and meta-analysis. Lancet Infect Dis 2011;11:845-54.

4) Alhazzani W, Smith O, Muscedere J, Medd J, Cook D. Toothbrushing for critically ill mechanically ventilated patients: a systematic review and meta-analysis of randomized trials evaluating ventilator-associated pneumonia. Crit Care Med 2013;41:646-55.

5) El-Solh AA, Pietrantoni C, Bhat A, Okada M, Zambon J, Aquilina A, et al. Colonization of dental plaques: a reservoir of respiratory pathogens for hospital-acquired pneumonia in institutionalized elders. Chest 2004;1261575-82.

6) Shay K, Scannapieco FA, Terpenning MS, Smith BJ, Taylor GW. Nosocomial pneumonia and oral health. Spec Care Dentist 2005;25:179-87.

7) Rello J, Diaz E. Pneumonia in the intensive care unit. Crit Care Med 2003:31:2544-51.

8) Brennan MT, Bahrani-Mougeot F, Fox PC, Kennedy TP, Hopkins S, Boucher RC, et al. The role of oral microbial colonization in ventilator-associated pneumonia. Oral Surg Oral Med Oral Pathol Oral Radiol Endod 2004;98:665-72.

9) Modi AR, Kovacs CS. Hospital-acquired and ventilator-associated pneumonia: Diagnosis, management, and prevention. Cleve Clin J Med 2020;87:633-9.

10) Kitsios GD, Fitch A, Manatakis DV, Rapport SF, Li K, Qin S, et al. Respiratory Microbiome Profiling for Etiologic Diagnosis of Pneumonia in Mechanically Ventilated Patients. Front Microbio/2018:9:1413.

11) Özçaka Ö, Başoğlu OK, Buduneli N, Taşbaken MS, Bacakoğlu F, Kinane DF. Chlorhexidine decreases the risk of ventilatorassociated pneumonia in intensive care unit patients: a randomized clinical trial. J Periodontal Res 2012;47:584-92.

12) Tuon FF, Gavrilko O, Almeida S, Sumi ER, Alberto T, Rocha JL, et al. Prospective, randomised, controlled study evaluating early modification of oral microbiota following admission to the intensive care unit and oral hygiene with chlorhexidine. J Glob Antimicrob Resist 2017;8:159-63.

13) Benson AK, Kelly SA, Legge R, Ma F, Low SJ, Kim J, et al. Individuality in gut microbiota composition is a complex polygenic trait shaped by multiple environmental and host genetic factors. Proc Nat/ Acad Sci U S A 2010;107:18933-8.

14) Deo PN, Deshmukh R. Oral microbiome: Unveiling the fundamentals. J Oral Maxillofac Patho/2019:23:122-8.

15) Somineni HK, Kugathasan S. The Microbiome in Patients With Inflammatory Diseases. Clin Gastroenterol Hepatol 2019;17:243-55.

16) Singh J. International conference on harmonization of technical requirements for registration of pharmaceuticals for human use. J Pharmacol Pharmacother 2015;6:185-7.

17) Bonham PA. Swab cultures for diagnosing wound infections: a literature review and clinical guideline. J Wound Ostomy Continence Nurs 2009;36:389-95.

18) Klindworth A, Pruesse E, Schweer T, Peplies J, Quast C, Horn M, et al. Evaluation of general 16 S ribosomal RNA gene PCR primers for classical and next-generation sequencing-based diversity studies. Nucleic Acids Res 2013;41:e1. 
19) Hall M, Beiko RG. 16S rRNA Gene Analysis with QIIME2. Methods Mol Bio/2018;1849:113-29.

20) Wade WG. The oral microbiome in health and disease. Pharmacol Res 2013;69:137-43.

21) Segata N, Izard J, Waldron L, Gevers D, Miropolsky L, Garrett WS, et al. Metagenomic biomarker discovery and explanation. Genome Bio/2011:12:R60.

22) Chastre J, Fagon JY. Ventilator-associated pneumonia. Am J Respir Crit Care Med 2002;165:867-903.

23) Bahrani-Mougeot FK, Paster BJ, Coleman S, Barbuto S, Brennan MT, Noll J, et al. Molecular analysis of oral and respiratory bacterial species associated with ventilator-associated pneumonia. J Clin Microbio/2007:45:1588-93.

24) Jakobsson HE, Jemberg C, Andersson AF, Sjölund-Karisson M, Jansson JK, Engstrand L. Short-term antibiotic treatment has differing long-term impacts on the human throat and gut microbiome. PloS one 2010;5:e9836.

25) Baek MG, Woo SJ, Kim NE, Baek C, Won S, Kim Y, et al. Respiratory microbiome profiles differ by recent hospitalization and nursing home residence in patients on mechanical ventilation. $J$ Trans/ Med 2020;18:464.

26) Harding CM, Hennon SW, Feldman MF. Uncovering the mechanisms of Acinetobacter baumannii virulence. Nat Rev Microbio/2018;16:91-102.

27) Song JH. Treatment recommendations of hospital-acquired pneumonia in Asian countries: first consensus report by the Asian HAP Working Group. Am J Infect Contro/2008;36:S84-92.

28) Kwak YG, Cho YK, Kim JR, Lee SO, Kim HY, Kim YK, et al. Korean Nosocomial Infections Surveillance System, Intensive Care Unit Module Report: Data Summary from July 2008 through June 2009 and Analysis of 3-Year Results. Korean J Nosocomial Infect Contro/2010;15:14-25. 